\title{
Análise dos fatores associados ao risco nutricional de pacientes em hemodiálise
}

\section{Analysis of factors associated with nutritional risk of patients on hemodialysis Análisis de los factores asociados con el riesgo nutricional de pacientes de hemodiálisis}

\author{
Jéssica Almeida Leite (iD \\ Universidade Federal de Mato Grosso - Cuiabá (MT) - Brasil \\ Priscila de Oliveira Sousa iD \\ Universidade Federal de Mato Grosso - Cuiabá (MT) - Brasil \\ Michelle Yasmine Borges (iD \\ Universidade Federal de Mato Grosso - Cuiabá (MT) - Brasil \\ Paulo Rogério Melo Rodrigues (iD \\ Universidade Federal de Mato Grosso - Cuiabá (MT) - Brasil \\ Bruna Teles Soares Beserra iD \\ Universidade Federal de Mato Grosso - Cuiabá (MT) - Brasil \\ Gabriela Dalcin Durante iD \\ Universidade Federal de Mato Grosso - Cuiabá (MT) - Brasil
}

\section{RESUMO}

Objetivo: Analisar a frequência e os fatores associados ao risco nutricional (RN) de pacientes com doença renal crônica (DRC) submetidos à hemodiálise. Métodos: Estudo de corte transversal, analítico, realizado com 110 indivíduos com idade $\geq 20$ anos, em tratamento hemodialítico em duas clínicas de Cuiabá, Mato Grosso, Brasil, no período de junho a outubro de 2018. Os dados foram coletados por meio de entrevista, aferições e consulta ao prontuário clínico. Avaliou-se a associação entre o desfecho pontuação do Malnutrition Inflammation Score (MIS) e as variáveis socioeconômicas, demográficas, clínicas, de comportamentos de risco relacionados à saúde e de estado nutricional. Realizou-se análise bivariada pelo teste de qui-quadrado de Pearson e regressão logística binária bruta e ajustada, considerando significativo $p \leq 0,05$. Resultados: Foi observada elevada frequência de indivíduos com alto $\mathrm{RN}$ ( $\mathrm{n}=73 ; 66,36 \%$ ), sendo maior entre os pacientes com tempo de diálise $\geq 4$ anos, volume de urina/24h $<500 \mathrm{ml}$, hiperpotassemia, hiperfosfatemia, fumantes, com elevada circunferência da cintura, baixa circunferência da panturrilha e que não praticavam exercício físico. $\mathrm{Na}$ análise múltipla ajustada permaneceram associados ao alto RN o maior tempo de diálise $(p=0,02)$ e o sedentarismo $(p=0,04)$. Conclusão: Observou-se elevada frequência de pacientes com alto RN, sendo que pacientes sedentários e com mais tempo de tratamento hemodialítico compuseram grupo em maior risco. Destaca-se a necessidade desses pacientes serem observados mais atentamente pelos profissionais de saúde que atuam nas clínicas de hemodiálise, a fim de promover melhor qualidade de vida e prevenir potenciais complicações e alterações do estado nutricional.

Descritores: Insuficiência Renal Crônica; Diálise Renal; Estado Nutricional.

\section{ABSTRACT}

Objective: To analyze the frequency and factors associated with nutritional risk (NR) in patients with Chronic Kidney Disease (CKD) undergoing hemodialysis. Methods: An analytical cross-sectional study was carried out with 110 individuals aged $\geq 20$ years undergoing hemodialysis in two clinics in Cuiabá, Mato Grosso, Brazil, from June to October 2018. Data were collected through interviews, measurements, and consultation on medical records. Associations between the outcome score on the Malnutrition Inflammation Score (MIS) and socioeconomic, demographic and clinical variables and health-related risk behaviors and nutritional status (NS) were assessed. Bivariate analysis was performed using Pearson's chi-squared test and crude and adjusted binary logistic regression were performed considering significant $p \leq 0.05$. Results: There was a high percentage of individuals with high NR ( $n=73 ; 66.36 \%)$, with higher rates among patients with dialysis duration $\geq 4$ years, 24-hour urine volume $<500 \mathrm{ml}$, hyperkalemia, hyperphosphatemia, smokers, those with increased waist circumference, low calf circumference and who did not practice physical exercises. In the adjusted multiple analysis, longer dialysis duration $(p=0.02)$ and physical inactivity

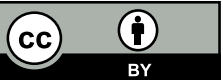


$(p=0.04)$ remained associated with high NR. Conclusion: There was a high frequency of patients with high NR, with sedentary patients and those with longer hemodialysis treatment being at a higher risk. There is a need for these patients to be observed more carefully by health professionals working in hemodialysis clinics in order to promote better quality of life and prevent potential complications and changes in nutritional status.

Descriptors: Renal Insufficiency, Chronic; Renal Dialysis; Nutritional Status.

\section{RESUMEN}

Objetivo: Analizar la frecuencia y los factores asociados con el riesgo nutricional $(R N)$ de pacientes con enfermedad renal crónica (ERC) sometidos a hemodiálisis. Métodos: Estudio de corte transversal y analítico realizado con 110 individuos de edad $\geq 20$ años, en tratamiento de hemodiálisis en dos clínicas de Cuiabá, Mato Grosso, Brasil, en el periodo entre junio y octubre de 2018. Se ha recogido los datos a través de entrevista, mediciones y consulta al historial clínico. Se evaluó la asociación entre el resultado de puntuación del Malnutrition Inflammation Score (MIS) y las variables socioeconómicas, demográficas, clínicas, de conductas de riesgo relacionadas con la salud y del estado nutricional. Se realizó un análisis bivariado con la prueba de chi-cuadrado de Pearson y la regresión logística binaria bruta y ajustada considerando significativo $p \leq 0,05$. Resultados: Se observó elevada frecuencia de individuos con alto $R N(n=73 ; 66,36 \%)$ que ha sido mayor entre los pacientes con el tiempo de diálisis $\geq 4$ años, el volumen de orina/ $24 \mathrm{~h}<500 \mathrm{ml}$, con hiperpotasemia y hiperfosfatemia, fumadores, con elevada circunferencia de la cintura, baja circunferencia de los gemelos y que no practicaban ejercicio físico. Más tiempo de diálisis $(p=0,02)$ y el sedentarismo $(p=0,04)$ se han asociado con el alto RN por el análisis múltiple ajustado. Conclusión: Se observó elevada frecuencia de pacientes con alto $R N$ y los pacientes sedentarios y con más tiempo de tratamiento de hemodiálisis han estado en el grupo de más riesgo. Se destaca la necesidad de los profesionales sanitarios que trabajan en las clínicas de hemodiálisis de observar a los pacientes más atentamente con el objetivo de promocionar mejor calidad de vida y la prevención de potenciales complicaciones y alteraciones del estado nutricional.

Descriptores: Insuficiencia Renal Crónica; Diálisis Renal; Estado Nutricional.

\section{INTRODUÇÃO}

O perfil epidemiológico global vem sofrendo mudanças, sendo estas resultantes da associação entre a redução do número total de mortes por doenças infectocontagiosas e aumento da ocorrência de mortalidade por doenças crônicas não transmissíveis (DCNT) $)^{(1)}$. Entre as DCNT, a doença renal crônica (DRC) tem grande impacto epidemiológico e é considerada um problema de saúde pública(2). Em 2019, a DRC apresentou posição de destaque entre as dez primeiras causas de anos de vida perdidos ajustados por incapacidade (Disability-adjusted Life Years - DALY) em grupos de idade mais avançada ${ }^{(2)}$.

A incidência e prevalência da DRC com necessidade de terapia renal substitutiva (TRS) vêm aumentando significativamente ao longo dos anos ${ }^{(2)}$. Segundo dados do Censo Brasileiro de Diálise ${ }^{(3)}$, a prevalência global estimada de pacientes em diálise crônica passou de 405 por milhão da população (pmp), em 2009, para 640 pmp em 2018 , correspondendo a um aumento absoluto de $58 \%$, com aumento médio de $6,4 \%$ ao ano. O número estimado de novos pacientes em diálise, em 2018, foi de 42.546, um aumento de $54,1 \%$ em relação a $2009^{(3)}$.

As principais doenças de base que resultam em DRC no Brasil são a hipertensão arterial (HA) e a diabetes mellitus (DM), e juntas representam cerca de 60 a $70 \%$ dos casos de DRC ${ }^{(3)}$. Essas três DCNT possuem em comum fatores de risco considerados modificáveis, tais como o excesso de peso, a inatividade física e alimentação inadequada. A alimentação adequada e saudável e as práticas corporais e atividade física são eixos prioritários da Política Nacional de Promoção da Saúde que têm impacto direto na prevenção da ocorrência de HA, DM e, como consequência, de $D R C^{(4)}$. A DRC caracteriza-se pela presença de dano renal ou redução das funções renais por um período igual ou superior a três meses, independente de sua etiologia, sendo a hemodiálise uma das alternativas de tratamento mais utilizadas nos indivíduos que necessitam de TRS ${ }^{(5)}$.

A redução da função renal e a própria TRS são responsáveis por provocar alterações no consumo alimentar e distúrbios hidroeletrolíticos, hormonais e metabólicos, que podem acarretar alterações no perfil nutricional resultando, principalmente, em depleção de gordura corporal e do tecido muscular ${ }^{(5,6)}$. A desnutrição possui grande impacto na evolução dos pacientes, uma vez que está associada a um maior número de complicações, maior frequência e duração de internações hospitalares, bem como maior mortalidade ${ }^{(7)}$.

Paradoxalmente, tem sido observado aumento da prevalência de sobrepeso e obesidade em pacientes com $\mathrm{DRC}^{(5)}$. Embora o excesso de peso seja identificado como uma causa importante da doença renal, devido a sua estreita associação com DM e HAS, e esteja associado a uma pior qualidade de vida para esses pacientes, visto que 
é considerado fator de risco para inúmeras doenças, ele tem sido considerado um fator de proteção em pacientes em diálise. Isto pode ocorrer pelo fato da desnutrição nesse grupo associar-se com maior mortalidade quando comparada aos indivíduos obesos ${ }^{(8)}$.

O estado nutricional (EN) dos pacientes renais crônicos tem grande impacto sobre sua qualidade de vida e sobrevida ${ }^{(5,6)}$, tornando-se essencial o seu monitoramento periódico, de forma a conhecer melhor as possíveis alterações nutricionais e metabólicas apresentadas, e atuar na promoção, manutenção ou recuperação da saúde desses indivíduos, minimizando seus danos ${ }^{(9,10)}$. Entre os métodos compostos de avaliação, o Malnutrition Inflammation Score (MIS) ${ }^{(11)}$ tem se destacado para a população com DRC por ser a única ferramenta que integra todos os métodos de avaliação do EN: história clínica, exame físico, antropometria e testes laboratoriais. Atualmente, o MIS é um avanço em relação às medidas individuais na avaliação do EN e vem sendo considerado um marcador clínico de risco nutricional na população em diálise ${ }^{(12)}$.

Dessa forma, o objetivo deste estudo é analisar a frequência e os fatores associados ao risco nutricional de pacientes com doença renal crônica submetidos à hemodiálise.

\section{MÉTODOS}

Trata-se de um estudo observacional, de corte transversal e analítico, com amostra não probabilística composta de 110 pacientes renais crônicos em tratamento hemodialítico em duas clínicas especializadas no município de Cuiabá, Mato Grosso, Brasil, no período de junho a outubro de 2018. As clínicas de hemodiálise eram particulares, porém atendiam majoritariamente pacientes do Sistema Único de Saúde (SUS) por meio de convênio.

Participaram da pesquisa todos os pacientes com DRC em hemodiálise que aceitaram e assinaram o Termo de Consentimento Livre e Esclarecido (TCLE), de ambos os sexos, com idade igual ou maior que 20 anos, em hemodiálise há pelo menos três meses, não hospitalizados nos últimos três meses, clinicamente estáveis e com prescrição de hemodiálise três ou mais vezes por semana. Excluíram-se da pesquisa pacientes gestantes, lactantes, com impossibilidade de realizar aferição de peso e/ou estatura, e pacientes com diagnóstico de comprometimento cognitivo e/ou de audição que poderia impedir a coleta de dados.

Realizou-se entrevista utilizando questionário próprio para obter as informações relacionadas às características socioeconômicas, demográficas e de comportamentos de risco relacionados à saúde, obtendo-se as variáveis clínicas por meio do prontuário clínico do paciente. Consideraram-se as seguintes variáveis demográficas: sexo (masculino e feminino); idade, em anos completos, categorizada em duas faixas etárias: 20-59 anos (adultos) e $\geq 60$ anos (idosos); raça/cor, autorreferida como branca, negra, parda, amarela ou vermelha e categorizada em brancos (raça/cor branca + amarela), pretos (raça/cor preta) e pardos (raça/cor parda + vermelha), devido à baixa frequência de indivíduos com ascendência oriental e indígena.

As variáveis socioeconômicas do estudo foram: escolaridade, coletada de acordo com período cursado, categorizada em: "até ensino fundamental completo" (incluindo indivíduos que referiram serem analfabetos ou ter cursado até o ensino médio incompleto), "ensino médio completo e superior incompleto" e "ensino superior completo ou mais" (incluindo aqueles com ensino superior completo e/ou qualquer nível de pós-graduação); situação conjugal, coletada conforme as opções solteiro(a), casado(a) legalmente, em união estável, viúvo(a), separado(a) ou divorciado(a), dicotomizada em "com companheiro(a)" ou "sem companheiro(a)" [solteiro(a), viúvo(a) ou separado(a)/divorciado(a)]; renda mensal individual, coletada conforme número de salários mínimos mensais individuais e categorizada em "até dois salários mínimos", "três ou mais salários mínimos"; e condição de trabalho atual, categorizada em "sem emprego" (desempregado), "trabalha" (incluindo autônomo, funcionário público ou de empresa privada) e "com renda, mas sem emprego" (incluindo aqueles que recebem auxílio-doença e aposentados por invalidez, tempo de serviço ou por idade).

As variáveis clínicas, autorreferidas ou coletadas no prontuário, são: volume de urina em 24 horas, coletado em mililitros e categorizada em "menor que $500 \mathrm{ml}$ " e "igual ou maior que $500 \mathrm{ml}$ "; tempo de diálise em meses, transformada para anos e dicotomizada em "menos que quatro anos" e "quatro anos ou mais". As comorbidades DM, HA e dislipidemia eram variáveis dicotômicas (sim/não).

Obtiveram-se os resultados dos exames laboratoriais a partir do prontuário clínico, sendo considerados os resultados de exames mais atuais em relação ao dia da coleta de dados. Os valores limítrofes para classificação dos resultados dos exames foram: ureia pré-diálise $\geq 150 \mathrm{mg} / \mathrm{dl}$ (valor esperado) ${ }^{(6)}$, fósforo e potássio $<5,5 \mathrm{mg} / \mathrm{dl}$ (valores esperados) ${ }^{(6)}$.

Em relação às variáveis de comportamento de risco relacionados à saúde, considerou-se as seguintes variáveis: fumante atual, variável dicotômica ( $\operatorname{sim} /$ não), sendo considerado fumante atual o indivíduo que respondeu positivamente à questão: "Atualmente, o(a) Sr.(a) é fumante?", independentemente do número de cigarros, da frequência e da duração do hábito de fumar; consumo de bebida alcoólica, variável dicotômica (sim/não), em resposta à questão: "O(a) Sr.(a) 
ingere algum tipo de bebida alcoólica?", independentemente da frequência de consumo, do tipo e quantidade de bebida alcoólica consumida; e prática de atividade física nos três meses anteriores à entrevista, variável dicotômica (sim/ não), em resposta à questão: "Nos últimos três meses, o(a) Sr.(a) praticou algum tipo de exercício físico ou esporte?", independentemente do tipo, frequência e tempo de duração do exercício físico ou esporte realizado.

Realizou-se a coleta de dados antropométricos e de exame físico ao término da sessão de hemodiálise para reduzir interferências do estado de hidratação do indivíduo. Para preenchimento do instrumento MIS, realizou-se o exame físico ${ }^{(10)}$ por meio de observação de perda aparente de tecido adiposo (classificando-as como: leve, moderada, grave, gravíssima ou não se aplica; observada nas regiões: bíceps, tríceps, peito, abaixo dos olhos) e muscular (classificando-as como: leve, moderada, grave, gravíssima ou não se aplica; observada nas regiões: têmpora, clavícula, acrômio, escápula, costelas músculo interósseo da mão, quadríceps, joelho e panturrilha).

A aferição das medidas antropométricas seguiu técnicas padronizadas, considerando: peso seco (peso após diálise $)^{(13)}$, altura ${ }^{(13)}$, circunferência da cintura $(C C)^{(14)}$, circunferência da panturrilha $(C P)^{(14)}$ e espessura do músculo adutor do polegar (EMAP) ${ }^{(15)}$. Realizaram-se, para cada medida, duas aferições em sequência, considerando-se como medida final a média das duas aferições.

A aferição do peso seco ocorreu por meio de balança digital modelo Ellegance (Mondial囚), com capacidade para $150 \mathrm{~kg}$. Os indivíduos estavam descalços, usando o mínimo de roupa possível e os braços estendidos ao longo do corpo ${ }^{(13)}$. Para aferição da altura, utilizou-se um estadiômetro compacto do tipo trena, marca Sanny®, com comprimento total de $210 \mathrm{~cm}$, e os participantes estavam em posição ereta, cabeça erguida, olhos mirando um plano horizontal de acordo com o plano horizontal de Frankfurt, com a coluna vertebral e calcanhares encostados na parede, joelhos esticados, pés juntos e braços estendidos ${ }^{(13)}$.

A partir do peso seco e da altura, calculou-se o Índice de Massa Corporal (IMC), sendo classificados, de acordo com a idade, em adultos ou idosos ${ }^{(13)}$. Ao final, categorizou-se a condição de peso em "sem excesso de peso" (incluindo todos aqueles indivíduos adultos e idosos classificados como baixo peso e eutrofia) ou "com excesso de peso".

Para aferição da CP e CC, utilizou-se fita métrica (marca Sanny ${ }^{\circledR}$, comprimento total de $150 \mathrm{~cm}$, com precisão de $1 \mathrm{~mm}$ ). A aferição da CP ocorreu no ponto de maior circunferência na panturrilha, sendo padronizada a aferição na perna esquerda ${ }^{(14)}$. Para essa medida, o participante permaneceu em posição ortostática ou sentado em cadeira com a perna flexionada a $90^{\circ}$. Para a CP, os indivíduos foram classificados em "risco nutricional", quando $\mathrm{CP}<31 \mathrm{~cm}$, ou "adequado", quando $\mathrm{CP} \geq 31 \mathrm{~cm}^{(14)}$.

Para a aferição da CC, o participante permaneceu em posição ortostática, os braços estendidos e levemente afastados do corpo e o abdômen relaxado. Convencionou-se a padronização da aferição no nível da cicatriz umbilical ${ }^{(14)}$. Para a CC, os indivíduos foram classificados em "risco baixo" ou "risco aumentado", considerando os valores limítrofes de CC associados ao desenvolvimento de complicações relacionadas à obesidade de acordo com o sexo ${ }^{(14)}$.

Realizou-se a aferição da EMAP com o paciente sentado, o braço flexionado a aproximadamente $90^{\circ}$, com o antebraço e a mão apoiados sobre o joelho ${ }^{(15)}$. Utilizou-se o plicômetro da marca Cescorf®, com pressão contínua de $10 \mathrm{~g} / \mathrm{mm}^{2}$, para pinçar o músculo adutor no vértice de um triângulo imaginário, formado pela extensão do polegar e do indicador. Realizou-se o procedimento na mão, do lado oposto à fistula arteriovenosa ${ }^{(16)}$. Quanto à medida da EMAP, os indivíduos foram classificados de acordo com valores médios de EMAP para pacientes em hemodiálise ${ }^{(16)}$, segundo o sexo, como "adequado" ou em "risco nutricional".

Ao final da coleta de dados antropométricos realizou-se o preenchimento do MIS. O instrumento contém 10 componentes, com níveis de gravidade pontuados de 0 a 3 . Os componentes avaliados são: história clínica (mudança de peso, apetite e ingestão alimentar, sintomas gastrointestinais, capacidade funcional e presença de comorbidades), exame físico (reservas gordurosa e muscular, e capacidade funcional), antropometria (IMC) e testes laboratoriais (albumina e capacidade total de ligação do ferro/transferrina) ${ }^{(10)}$. A soma de todos os componentes pode variar de 0 a 30 . A classificação do risco nutricional, segundo o MIS, consistiu em baixo (<6 pontos) ou alto risco nutricional ( $\geq 6$ pontos) ${ }^{(17)}$.

Os dados, digitados em duplicata no programa Epilnfo, versão 7.2, passaram por análise de consistência por meio de comparação entre as digitações para eliminar possíveis erros, sendo armazenados em banco de dados Excel e depois analisados utilizando o pacote estatístico SPSS, versão 23.0 (SPSS Inc. Chicago IL, USA).

Para verificar a consistência dos dados e definir as categorias das variáveis, estimaram-se as frequências absolutas e relativas das variáveis. Utilizou-se o teste qui-quadrado de Pearson para a análise bivariada e, quando necessário, também o teste exato de Fisher. Incluíram-se as variáveis independentes que apresentaram $p \leq 0,10$ na análise bivariada por meio do teste de Pearson no modelo de regressão logística binária bruta e ajustada mutuamente por todas as variáveis do modelo. $\mathrm{Na}$ análise ajustada, foram utilizadas todas as covariáveis analisadas, sendo as covariáveis menos informativas sucessivamente removidas do modelo, em uma eliminação backward com 
base no critério de informação de Akaike. Aquelas variáveis independentes que permaneceram com $p \leq 0,05$ foram consideradas associadas à variável desfecho MIS e mantidas no modelo múltiplo final.

Este estudo obteve a aprovação da direção das clínicas, locais de realização do estudo, e do Comitê de Ética em Pesquisa com Seres Humanos da Universidade Federal de Mato Grosso (Parecer n. ${ }^{\circ}$ 2.658.607).

\section{RESULTADOS}

Ao todo, participaram 110 pacientes, com média de idade de 51,8 anos (desvio-padrão $=12,8$ ). Verificou-se que a maioria da população era do sexo masculino $(n=68 ; 61,82 \%)$, com predominância de indivíduos adultos (entre 20 e 59 anos) ( $n=75 ; 68,19 \%)$, autorreferidos da raça/cor parda ( $n=60 ; 54,54 \%)$ e que vivem com companheiro(a) $(n=59 ; 53,64 \%)$. Quanto à escolaridade, constatou-se que 47,22\% $(n=51)$ dos pacientes possuíam apenas o ensino fundamental completo. A maioria dos participantes é beneficiária da previdência social $(n=90 ; 82,57 \%)$ e a renda média individual era de até dois salários mínimos ( $n=78 ; 70,91 \%$ ) (Tabela I).

Do total de pacientes avaliados, 66,36\% $(n=73)$ apresentaram MIS igual ou maior que 6 pontos, sendo a pontuação média apresentada pelos pacientes deste estudo de 6,99 (desvio-padrão $=2,97$ ). Entre as variáveis socioeconômicas e demográficas não foram verificadas diferenças estatisticamente significativas segundo as categorias de MIS (Tabela I).

Tabela I - Características socioeconômicas e demográficas e análise bivariada entre as categorias do Malnutrition Inflammation Score (MIS) e variáveis socioeconômicas e demográficas de pacientes em tratamento hemodialítico em duas clínicas. Cuiabá, Mato Grosso, Brasil, 2018.

\begin{tabular}{|c|c|c|c|c|c|c|c|}
\hline \multirow{3}{*}{ Variáveis } & \multicolumn{2}{|c|}{ Total $(n=110)$} & \multicolumn{4}{|c|}{ MIS } & \multirow{3}{*}{ p-valor } \\
\hline & \multirow[b]{2}{*}{$\mathbf{n}$} & \multirow[b]{2}{*}{$\%$} & \multicolumn{2}{|c|}{$\begin{array}{l}\text { Risco baixo } \\
\text { (<6 pontos) }\end{array}$} & \multicolumn{2}{|c|}{$\begin{array}{l}\text { Risco alto } \\
\text { ( } \geq 6 \text { pontos) }\end{array}$} & \\
\hline & & & $\mathbf{n}$ & $\%$ & $\mathbf{n}$ & $\%$ & \\
\hline Total & 110 & 100 & 37 & 33,64 & 73 & 66,36 & \\
\hline Sexo & & & & & & & $0,96^{\mathrm{a}}$ \\
\hline Feminino & 42 & 38,18 & 14 & 33,33 & 28 & 66,67 & \\
\hline Masculino & 68 & 61,82 & 23 & 33,82 & 45 & 66,18 & \\
\hline Faixa etária & & & & & & & $0,23^{\mathrm{a}}$ \\
\hline Adulto & 75 & 68,19 & 28 & 37,33 & 47 & 62,67 & \\
\hline Idoso & 35 & 31,81 & 9 & 25,71 & 26 & 74,29 & \\
\hline Raça/cor & & & & & & & $0,58^{\mathrm{a}}$ \\
\hline Branca & 23 & 20,91 & 9 & 39,13 & 14 & 60,87 & \\
\hline Preta & 27 & 24,55 & 7 & 25,93 & 20 & 74,07 & \\
\hline Parda & 60 & 54,54 & 21 & 35,00 & 39 & 65,00 & \\
\hline Escolaridade & & & & & & & $0,16^{\mathrm{a}}$ \\
\hline Até ens. fundamental completo & 51 & 47,22 & 13 & 25,49 & 38 & 74,51 & \\
\hline Ens. médio completo e superior incompleto & 41 & 37,96 & 15 & 36,59 & 26 & 63,41 & \\
\hline Ens. superior completo ou mais & 16 & 14,82 & 8 & 50,00 & 8 & 50,00 & \\
\hline Situação conjugal & & & & & & & $0,38^{\mathrm{a}}$ \\
\hline Sem companheiro(a) & 51 & 46,36 & 15 & 29,41 & 36 & 70,59 & \\
\hline Com companheiro(a) & 59 & 53,64 & 22 & 37,29 & 37 & 62,71 & \\
\hline Renda individual mensal & & & & & & & $0,32^{\mathrm{a}}$ \\
\hline Até dois salários & 78 & 70,91 & 24 & 30,77 & 54 & 69,23 & \\
\hline Três ou mais salários & 32 & 29,09 & 13 & 40,63 & 19 & 59,37 & \\
\hline Condição de trabalho & & & & & & & $0,67^{b}$ \\
\hline Sem emprego & 8 & 7,34 & 3 & 37,50 & 5 & 62,50 & \\
\hline Trabalha & 11 & 10,09 & 5 & 45,45 & 6 & 54,55 & \\
\hline Com renda, mas sem emprego & 90 & 82,57 & 29 & 32,22 & 61 & 67,78 & \\
\hline
\end{tabular}

a: teste de qui-quadrado de Pearson; b: teste exato de Fisher, $\mathrm{p} \leq 0,05$; ens.: ensino 
As etiologias presuntivas mais frequentes de DRC foram: a nefroesclerose hipertensiva ( $n=54 ; 49,09 \%)$, causas desconhecidas ou não diagnosticadas $(n=25 ; 22,73 \%)$ e a nefropatia diabética (DRC causada pela diabetes mellitus) $(n=12 ; 10,91 \%)$ (dados não apresentados em tabela). As características clínicas dos pacientes são apresentadas na Tabela II, bem como a análise bivariada entre essas variáveis e as categorias do MIS.

Em relação à presença de comorbidades, a $\mathrm{HA}(n=83 ; 75,45 \%)$ e o $\mathrm{DM}(\mathrm{n}=26 ; 23,64 \%)$ foram frequentes nessa população. Quanto ao tempo de diálise, a média, em anos, foi 4,86 (desvio-padrão $=4,65$ ) e a maioria dos pacientes apresentou tempo de tratamento menor que quatro anos ( $n=63 ; 57,27 \%)$.

$\mathrm{Na}$ análise bivariada entre as variáveis do MIS e as variáveis clínicas, observou-se que, para o tempo de diálise, a proporção de indivíduos com alto risco foi maior entre aqueles com quatro anos ou mais em comparação àqueles que tinham menos de quatro anos de tratamento hemodialítico $(n=38 ; 80,85 \%$ versus $n=35 ; 55,56 \% ; p=0,01)$. A maioria dos pacientes apresentou o volume residual de diurese menor que $500 \mathrm{ml}$ no período de 24 horas $(\mathrm{n}=84$; $76,36 \%)$, sendo denominados anúricos e/ou oligúricos, estando essa categoria associada a maior risco nutricional $(n=61 ; 72,62 \% ; p=0,01)$.

Tabela II - Características clínicas e análise bivariada entre as categorias do Malnutrition Inflammation Score (MIS) e as variáveis clínicas de pacientes em tratamento. Cuiabá, Mato Grosso, Brasil, 2018.

\begin{tabular}{|c|c|c|c|c|c|c|c|}
\hline \multirow{3}{*}{ Variáveis } & \multicolumn{2}{|c|}{ Total $(n=110)$} & \multicolumn{4}{|c|}{ MIS } & \multirow{3}{*}{ p-valor } \\
\hline & \multirow[b]{2}{*}{$\mathrm{n}$} & \multirow[b]{2}{*}{$\%$} & \multicolumn{2}{|c|}{$\begin{array}{l}\text { Risco baixo } \\
\text { ( } \leq 6 \text { pontos) }\end{array}$} & \multicolumn{2}{|c|}{$\begin{array}{l}\text { Risco alto } \\
\text { ( } \geq 6 \text { pontos) }\end{array}$} & \\
\hline & & & $\mathbf{n}$ & $\%$ & $\mathbf{n}$ & $\%$ & \\
\hline Diabetes & & & & & & & $0,41^{a}$ \\
\hline Sim & 26 & 23,64 & 7 & 26,92 & 19 & 73,08 & \\
\hline Não & 84 & 76,36 & 30 & 35,71 & 54 & 64,29 & \\
\hline Hipertensão arterial & & & & & & & $0,67^{a}$ \\
\hline $\operatorname{Sim}$ & 83 & 75,45 & 27 & 32,53 & 56 & 67,47 & \\
\hline Não & 27 & 24,55 & 10 & 37,04 & 17 & 62.96 & \\
\hline Dislipidemia & & & & & & & $0,73^{b}$ \\
\hline Sim & 10 & 9,09 & 4 & 40,00 & 6 & 60,00 & \\
\hline Não & 100 & 90,91 & 33 & 33,00 & 67 & 67,00 & \\
\hline Tempo de diálise & & & & & & & $0,01^{a^{*}}$ \\
\hline Menos que quatro anos & 63 & 57,27 & 28 & 44,44 & 35 & 55,56 & \\
\hline Quatro anos ou mais & 47 & 42,73 & 9 & 19,15 & 38 & 80,85 & \\
\hline Volume de urina/24h & & & & & & & $0,01^{a^{*}}$ \\
\hline$<500 \mathrm{ml}$ & 84 & 76,36 & 23 & 27,38 & 61 & 72,62 & \\
\hline$\geq 500 \mathrm{ml}$ & 26 & 23,64 & 14 & 53,85 & 12 & 46,15 & \\
\hline Ureia pré-diálise & & & & & & & $0,06^{a}$ \\
\hline Abaixo do valor esperado & 70 & 63,64 & 28 & 40,00 & 42 & 60,00 & \\
\hline Valor esperado & 40 & 36,36 & 9 & 22,50 & 31 & 77,50 & \\
\hline Potássio & & & & & & & $0,09^{a}$ \\
\hline Valor esperado & 78 & 70,91 & 30 & 38,46 & 48 & 61,54 & \\
\hline Acima do valor esperado & 32 & 29,09 & 7 & 21,88 & 25 & 78,12 & \\
\hline Fósforo & & & & & & & $0,07^{a}$ \\
\hline Valor esperado & 77 & 70,00 & 30 & 39,00 & 47 & 61,00 & \\
\hline Acima do valor esperado & 33 & 30,00 & 7 & 21,21 & 26 & 78,79 & \\
\hline
\end{tabular}

a: teste de qui-quadrado de Pearson; ${ }^{\text {b: }}$ teste exato de Fisher, $\mathrm{p} \leq 0,05$

Os comportamentos de risco relacionado à saúde e ao estado nutricional dos pacientes em hemodiálise, segundo as categorias do MIS, estão descritos na Tabela III. O tabagismo não é uma prática frequente na população analisada $(n=7 ; 6,36 \%)$, porém $19,27 \%(n=21)$ referiu consumir bebida alcoólica. Do total de pacientes, $62,73 \%(n=69)$ eram 
sedentários, e dos pacientes que referiram praticar algum tipo de exercício físico ( $n=41 ; 37,27 \%)$ nos últimos três meses anteriores à entrevista, a caminhada foi o mais relatado $(n=27 ; 66,36 \%)$.

A respeito do estado nutricional dos pacientes, verificou-se que $45,45 \%(n=50)$ apresentaram algum grau de excesso de peso segundo o IMC, e a maioria $(n=73 ; 66,36 \%)$ apresentou risco aumentado para doenças cardiovasculares (DCV) segundo a medida de CC. Quanto aos indicadores de massa muscular, a maioria dos pacientes apresentou valores adequados para $\mathrm{CP}(\mathrm{n}=91 ; 82,73 \%)$ e frequências semelhantes para as categorias "adequado" e "risco nutricional" da EMAP ( $n=55 ; 50,00 \%)$. Na análise bivariada, observou-se que uma maior proporção de indivíduos com risco nutricional segundo a CP foi observada entre os que apresentavam risco alto segundo o MIS $(n=17$; 89,47\%; $p=0,02$ ) (Tabela III).

$\mathrm{Na}$ Tabela IV são apresentados os resultados da análise múltipla bruta e ajustada, sendo observado que o sedentarismo $(p=0,02)$ e o maior tempo de diálise $(p=0,04)$ foram associados ao alto risco nutricional (MIS $\geq 6$ pontos). Em relação à prática de exercício físico nos três meses anteriores à entrevista, aqueles indivíduos que referiram não praticar apresentaram 3,25 vezes mais chances de ter maior risco nutricional quando comparados aos indivíduos que relataram praticar algum exercício físico. Quanto ao tempo de diálise, os indivíduos com quatro anos ou mais de tratamento apresentaram 3,03 vezes mais chances de ter maior risco nutricional em comparação àqueles indivíduos com menos de quatro anos de hemodiálise.

Tabela III - Características de comportamentos de risco relacionados à saúde e do estado nutricional de pacientes e análise bivariada entre as categorias do Malnutrition Inflammation Score (MIS) e variáveis de comportamentos de risco relacionados à saúde e do estado nutricional de pacientes em tratamento. Cuiabá, Mato Grosso, Brasil, 2018.

\begin{tabular}{|c|c|c|c|c|c|c|c|}
\hline \multirow{3}{*}{ Variáveis } & \multicolumn{2}{|c|}{ Total $(n=110)$} & \multicolumn{4}{|c|}{ MIS } & \multirow{3}{*}{ p-valor } \\
\hline & \multirow[b]{2}{*}{$n$} & \multirow[b]{2}{*}{$\%$} & \multicolumn{2}{|c|}{$\begin{array}{l}\text { Risco baixo } \\
\text { (<6 pontos) }\end{array}$} & \multicolumn{2}{|c|}{$\begin{array}{l}\text { Risco alto } \\
\text { ( } \geq 6 \text { pontos) }\end{array}$} & \\
\hline & & & $\mathbf{n}$ & $\%$ & $\mathbf{n}$ & $\%$ & \\
\hline Fumante atual & & & & & & & $0,09^{b}$ \\
\hline Sim & 7 & 6,36 & 0 & 0,00 & 7 & 100,00 & \\
\hline Não & 103 & 93,64 & 37 & 35,92 & 66 & 64,08 & \\
\hline Consumo de bebida alcoólica & & & & & & & $0,65^{\mathrm{a}}$ \\
\hline Não & 21 & 19,27 & 8 & 38,10 & 13 & 61,90 & \\
\hline Sim & 88 & 80,73 & 29 & 32,95 & 59 & 67,05 & \\
\hline Prática de exercício físico & & & & & & & $0,08^{\mathrm{a}}$ \\
\hline Sim & 41 & 37,27 & 18 & 43,90 & 23 & 56,10 & \\
\hline Não & 69 & 62,73 & 19 & 27,54 & 50 & 72,46 & \\
\hline Condição de peso & & & & & & & $0,20^{\mathrm{a}}$ \\
\hline Sem excesso de peso & 60 & 54,55 & 17 & 28,33 & 43 & 71,67 & \\
\hline Com excesso de peso & 50 & 45,45 & 20 & 40,00 & 30 & 60,00 & \\
\hline Circunferência da cintura & & & & & & & $0,06^{\mathrm{a}}$ \\
\hline Risco baixo & 37 & 33,64 & 8 & 21,62 & 29 & 78,38 & \\
\hline Risco aumentado & 73 & 66,36 & 29 & 39,73 & 44 & 60,27 & \\
\hline Circunferência da panturrilha & & & & & & & $0,02^{a^{*}}$ \\
\hline Risco nutricional & 19 & 17,27 & 2 & 10,53 & 17 & 89,47 & \\
\hline Adequado & 91 & 82,73 & 35 & 38,46 & 56 & 61,54 & \\
\hline EMAP & & & & & & & $0,07^{\mathrm{a}}$ \\
\hline Risco nutricional & 55 & 50,00 & 14 & 25,45 & 41 & 74,55 & \\
\hline Adequado & 55 & 50,00 & 23 & 41,82 & 32 & 58,18 & \\
\hline
\end{tabular}

EMAP: Espessura do músculo adutor do polegar; a: teste de qui-quadrado de Pearson; ${ }^{\mathrm{b}}$ : teste exato de Fisher; $\mathrm{p} \leq 0,05$ 
Tabela IV - Regressão logística binária bruta e ajustada entre o Malnutrition Inflammation Score (MIS) e variáveis independentes de pacientes em tratamento. Cuiabá, Mato Grosso, Brasil, 2018.

\begin{tabular}{|c|c|c|c|c|c|c|}
\hline \multirow{3}{*}{ Variáveis } & \multicolumn{6}{|c|}{ MIS } \\
\hline & \multicolumn{3}{|c|}{ Análise bruta } & \multicolumn{3}{|c|}{ Análise ajustada* } \\
\hline & OR & (IC 95\%) & p-valor & OR & (IC 95\%) & p-valor \\
\hline Prática de exercício físico & & & 0,08 & & & $0,02^{* *}$ \\
\hline Não & 2,06 & 0,$91 ; 4,64$ & & 3,25 & 1,$23 ; 8,73$ & \\
\hline Sim & 1,00 & & & 1,00 & & \\
\hline Circunferência da cintura & & & 0,06 & & & - \\
\hline Baixo risco & 2,39 & 0,$96 ; 5,95$ & & - & - & \\
\hline Risco aumentado & 1,00 & & & - & - & \\
\hline Circunferência da panturrilha & & & $0,03^{* *}$ & & & \\
\hline Risco nutricional & 5,31 & 1,$16 ; 24,41$ & & - & - & \\
\hline Adequado & 1,00 & & & - & - & \\
\hline EMAP & & & 0,07 & & & - \\
\hline Risco nutricional & 2,10 & 0,$94 ; 4,73$ & & - & - & \\
\hline Adequado & 1,00 & & & - & - & \\
\hline Tempo de diálise & & & $0,01^{* *}$ & & & $0,04^{* *}$ \\
\hline Menos que 4 anos & 1,00 & & & 1,00 & & \\
\hline 4 ou mais anos & 3,38 & 1,$40 ; 8,15$ & & 3,03 & 1,$03 ; 8,90$ & \\
\hline Volume de urina/24h & & & $0,01^{* *}$ & & & - \\
\hline$<500 \mathrm{ml}$ & 3,09 & 1,$25 ; 7,67$ & & - & - & \\
\hline$\geq 500 \mathrm{ml}$ & 1,00 & & & - & - & \\
\hline Ureia pré-diálise & & & 0,06 & & & - \\
\hline Abaixo do valor esperado & 0,44 & 0,$18 ; 1,05$ & & - & - & \\
\hline Valor esperado & 1,00 & & & - & - & \\
\hline Potássio & & & 0,10 & & & - \\
\hline Valor esperado & 0,45 & 0,$17 ; 1,16$ & & - & - & \\
\hline Acima do valor esperado & 1,00 & & & - & - & \\
\hline Fósforo & & & 0,08 & & & - \\
\hline Valor esperado & 0,42 & 0,$16 ; 1,09$ & & - & - & \\
\hline Acima do valor esperado & 1,00 & & & - & - & \\
\hline
\end{tabular}

OR: Odds ratio; IC 95\%: Intervalo de confiança de 95\%; EMAP: Espessura do músculo adutor do polegar; *Análise mutuamente ajustada por todas as variáveis do modelo; ${ }^{* *} p<0,05$

\section{DISCUSSÃO}

No presente estudo foram avaliados os fatores associados ao risco nutricional em pacientes em tratamento hemodialítico, constatando-se elevada frequência de indivíduos com alto risco nutricional, sendo a maior entre os pacientes com tempo de diálise $\geq 4$ anos, volume de urina $/ 24 \mathrm{~h}<500 \mathrm{ml}$, hiperpotassemia, hiperfosfatemia, fumantes, com elevada circunferência da cintura, baixa circunferência da panturrilha e que não praticavam exercício físico. $\mathrm{Na}$ análise múltipla ajustada, aqueles indivíduos sedentários e que possuíam maior tempo de diálise apresentaram maior risco nutricional, segundo o MIS, quando comparados àqueles praticantes de exercício físico e com menor tempo em HD.

As características socioeconômicas e demográficas dos participantes avaliados no atual estudo foram semelhantes àquelas encontradas em outros estudos nacionais em que a maioria dos pacientes com DRC é do sexo masculino ${ }^{(18,19)}$, de raça/cor parda ${ }^{(18,19)}$, que vivem com companheiro(a) $)^{(20)}$, com baixo nível socioeconômico ${ }^{(19,20)}$ e de escolaridade ${ }^{(18,19)}$. Quanto às etiologias presuntivas mais frequentes de DRC, verificou-se que a nefroesclerose hipertensiva e a nefropatia diabética, juntas, somaram mais da metade dos casos encontrados. Além disso, a HA e o DM foram as comorbidades mais frequentes nessa população. 
No Brasil, HA e DM são as doenças de base e comorbidades mais frequentes associadas à DRC ${ }^{(4)}$. A ocorrência dessas DCNT segue a tendência atual do perfil geral da população mundial, em que há um aumento das DCNT e seus fatores de risco em comum modificáveis, tais como alimentação não saudável, inatividade física, tabagismo, consumo nocivo de bebidas alcoólicas e excesso de peso(2,21). Esses fatores de risco fazem parte do plano de enfrentamento de DCNT 20112022(21), e seu controle tem impacto direto na incidência e na prevalência de DRC.

A avaliação do EN por meio do instrumento MIS ainda não possui um ponto de corte consolidado para pacientes em hemodiálise. Além disso, em estudos realizados com essa população, podem ser encontrados diferentes pontos de corte para classificar maior risco nutricional|(22,23). No presente estudo, os participantes com pontuação igual ou maior que seis pontos no MIS foram classificados com pior condição nutricional. A pontuação média observada para o MIS foi semelhante aos valores encontrados em outros estudos realizados com pacientes renais crônicos pelo mundo ${ }^{(18,23)}$.

A maioria dos pacientes foi considerada anúrica ou oligúrica na atual pesquisa, sendo essa condição associada a um maior risco nutricional segundo o MIS, porque o volume residual da diurese auxilia na depuração de substâncias de baixo e médio pesos moleculares, assim permitindo maior remoção de fluidos e melhora do $\mathrm{EN}^{(24)}$. Além disso, o volume residual determina quanto o paciente deve restringir sua alimentação e ingestão de líquidos ${ }^{(24)}$. Pode-se deduzir que pacientes com menor volume urinário apresentam maiores restrições alimentares e, desse modo, 0 EN pode ser afetado negativamente(25).

A maior parte dos pacientes da atual pesquisa apresentou valores aumentados dos níveis séricos de fósforo e potássio no estudo em questão, e essa condição está associada ao maior risco nutricional, visto que a hiperfosfatemia contribui para o hiperparatireoidismo secundário, a resistência à vitamina $D$ e a hipocalcemia, além de ser responsável por provocar danos aos ossos e risco aumentado para $\mathrm{DCV}^{(6)}$, e a hiperpotassemia pode levar à arritmia cardíaca e morte súbita, particularmente os pacientes em hemodiálise ${ }^{(5)}$.

Quanto ao estado nutricional da população analisada nesta pesquisa, verificou-se que em torno de $45 \%$ dos participantes apresentaram algum grau de excesso de peso segundo o IMC e a maioria apresentou risco aumentado de desenvolvimento de DCV segundo a CC. Esses dados seguem a tendência atual da população em geral e da população renal crônica( ${ }^{(8)}$. Em pacientes renais crônicos tem sido relatado que o IMC elevado é, paradoxalmente, associado à melhor sobrevivência nesses pacientes, um fenômeno referido como epidemiologia reversa, talvez porque, nesse mesmo grupo, a desnutrição associa-se com maior mortalidade quando comparada aos indivíduos obesos $^{(8)}$. Entretanto, mesmo nos pacientes em diálise, a obesidade visceral é associada com aumento do risco cardiovascular, especialmente risco de calcificações coronarianas e outros eventos cardiovasculares adversos ${ }^{(8)}$.

Observou-se, ainda no presente estudo, que a maioria dos indivíduos com menor espessura do músculo adutor do polegar (considerado risco nutricional) apresentou também maior pontuação no MIS (risco alto), demonstrando que essa medida pode ser indicadora de redução da massa muscular e de alteração da composição corporal, embora os valores de referência utilizados para categorização das medidas da EMAP não sejam unânimes entre as publicações sobre a população renal crônica ${ }^{(16,26)}$.

Destaca-se que, na análise múltipla, permaneceram associados ao alto risco nutricional o sedentarismo e maior tempo de tratamento hemodialítico dos participantes analisados no atual estudo. Em relação à prática de exercício físico, indivíduos sedentários apresentaram 225\% mais chances de ter maior risco nutricional quando comparadas aos praticantes. Não foram encontrados na literatura estudos que analisaram a associação entre a prática de exercício físico e o MIS, porém o sedentarismo tem sido observado entre pacientes com DRC, e isto pode ser associado ao aumento da mortalidade, morbidade e aos fatores de risco que impulsionam a progressão da DRC ${ }^{(27)}$. Como consequência, entre pacientes renais crônicos, o sedentarismo pode resultar em redução da massa muscular (sarcopenia), redução da força esquelética e no acúmulo de tecido adiposo, situação agravada devido às alterações da composição corporal provocadas pela própria DRC e pela TRS, o que pode acarretar a desnutrição e o declínio da capacidade funcional desses pacientes ${ }^{(25,27,28)}$.

O exercício físico promove inúmeros benefícios, como controle dos níveis glicêmicos e a redução da pressão arterial, com efeitos adicionais sobre o controle de peso, a melhora do perfil lipídico, o fortalecimento dos ossos e dos músculos esqueléticos, a melhoria do estado nutricional e, consequentemente, melhoria da qualidade de vida $^{(25,27,28)}$. Assim como para a população em geral, indivíduos com DRC são beneficiados pelas ações da Política Nacional de Promoção da Saúde ${ }^{(4)}$ ao elencar, entre seus temas prioritários, as práticas corporais e atividades físicas, porque mesmo em treinamento de menor intensidade, o exercício físico pode resultar no controle e reversão da perda muscular e na melhoria da capacidade funcional de pacientes renais crônicos ${ }^{(27)}$.

Quanto ao tempo de diálise, encontrou-se no atual estudo que os indivíduos com quatro anos ou mais de tratamento hemodialítico apresentaram $203 \%$ vezes mais chances de ter maior risco nutricional em comparação 
àqueles com menos de quatro anos de tratamento. Corroborando esse achado do presente estudo, outras publicações sugerem que quanto maior o tempo em tratamento hemodialítico, maior a pontuação do MIS, assinalando um pior estado nutricional ${ }^{(22,29)}$. A associação entre o tempo de tratamento hemodialítico e o MIS pode ser explicada pela inflamação subclínica e a redução da síntese proteica, pela redução da massa muscular e alteração da composição corporal em resposta à progressão da DRC, e pelas alterações metabólicas provocadas pela própria TRS ${ }^{(22,24,29)}$.

A fim de minimizar o impacto da DRC e de seu tratamento sobre o estado nutricional do paciente, recomendam-se que sejam realizadas avaliações nutricionais de rotina ${ }^{(9,10)}$. Sabe-se que não há um método único que pode predizer desnutrição em hemodiálise e também que a indicação mais acurada é o uso de múltiplos métodos aplicados simultaneamente $^{(10,16)}$. O MIS tem se destacado como um instrumento composto de avaliação do EN de pacientes em hemodiálise e como importante marcador clínico nessa população ${ }^{(12)}$, podendo diagnosticar precocemente alterações do estado nutricional. Além disso, ações de promoção de hábitos de vida saudáveis, como alimentação adequada e saudável, práticas corporais e atividades físicas, cessação do tabagismo e do consumo de bebidas alcoólicas ${ }^{(4)}$, contribuem em curto, médio e longo prazo para a melhoria do estado nutricional e a qualidade de vida de pacientes em hemodiálise.

Uma limitação do presente estudo é o fato dos exames laboratoriais terem sido coletados em duas clínicas diferentes e com dados coletados no prontuário dos pacientes, de modo que não houve padronização das datas de coleta e dos laboratórios de análise. Porém adotou-se como prática a coleta dos dados mais atuais disponíveis nos prontuários. Além disso, o exame físico contido no instrumento MIS baseia-se em uma avaliação subjetiva da diminuição da reserva de gordura corporal e subcutânea, bem como de sinais de redução da massa muscular, e essa avaliação pode diferir entre avaliadores. Para minimizar esse erro, adotou-se como procedimento o treinamento para coleta de dados e a padronização da avaliação realizada pelo pesquisador que realizava essa análise.

\section{CONCLUSÃO}

Observou-se elevada frequência de pacientes com alto $\mathrm{RN}$, sendo que pacientes sedentários e com mais tempo de tratamento hemodialítico compuseram o grupo com maior risco nutricional segundo o instrumento MIS. Destaca-se a necessidade desses pacientes serem observados mais atentamente pelos profissionais de saúde que atuam nas clínicas de hemodiálise, a fim de promover melhor qualidade de vida e prevenir potenciais complicações e alterações do estado nutricional.

\section{CONFLITOS DE INTERESSE}

Os autores declaram a inexistência de conflitos de interesses.

\section{CONTRIBUIÇÕES DOS AUTORES}

Jéssica Almeida Leite, Priscila Oliveira Sousa, Michelle Yasmine Borges e Gabriela Dalcin Durante participaram de todas as etapas da pesquisa, com contribuições substanciais na concepção e desenho do estudo; na coleta, análise e interpretação dos dados; e na redação e revisão crítica do artigo. Bruna Teles Soares Beserra participou da análise e interpretação dos dados; e da revisão crítica do artigo. Paulo Rogério Melo Rodrigues contribuiu na análise estatística e interpretação dos dados, bem como na revisão crítica do artigo. Todos os autores aprovaram a versão final submetida à avaliação.

\section{REFERÊNCIAS}

1. World Health Organization. World Health Statistics 2018: monitoring health for the SDGs, sustainable development goals. Geneva: World Health Organization; 2018.

2. Global Burden of Disease 2016 Causes of Death Collaborators. Global, regional, and national age-sex specific mortality for 264 causes of death, 1980-2016: a systematic analysis for the Global Burden of Disease Study 2016. Lancet [Internet]. 2017 [acesso em 2020 Nov 12];390(10100):1151-210. doi: https://doi. org/10.1016/S0140-6736(17)32152-9

3. Neves PDMM, Sesso RCC, Thomé FS, Lugon JR, Nascimento MM. Censo Brasileiro de Diálise: análise dos dados da década de 2009-2018. J Bras Nefrol [Internet]. 2020 [acesso em 2020 Nov 12];42(2):191-200. doi: 10.1590/2175-8239-JBN-2019-0234 
4. Brasil. Ministério da Saúde. Portaria $n^{\circ} 2.446$, de 11 de novembro de 2014. Redefine a Política Nacional de Promoção da Saúde (PNPS). Diário Oficial da União; 2014.

5. Avesani CM, Pereira AML, Cuppari L. Doença renal crônica. In: Cuppari L. Nutrição nas doenças crônicas não transmissíveis. Barueri: Manole; 2009. p. 267-330.

6. Martins C. Avaliação e diagnóstico do estado nutricional de pacientes renais. In: Riella MC, Martins C. Nutrição e o rim. Rio de Janeiro: Guanabara Koogan; 2013. p. 93-109.

7. Bousquet-Santos K, Costa LG, Andrade JML. Estado nutricional de portadores de doença renal crônica em hemodiálise no Sistema Único de Saúde. Ciênc Saúde Colet [Internet]. 2019 [acesso em 2020 Nov 12];(3):1189-99. doi: 10.1590/1413-81232018243.11192017

8. Silva GB Jr, Bentes ACSN, Daher EF, Matos SMA. Obesidade e doença renal. J Bras Nefrol [Internet]. 2017 [acesso em 2020 Nov 12];39(1):65-69. doi:10.5935/0101-2800.20170011

9. Silva AMD, Souto TCM, Freitas FF, Moraes CN, Sousa BS. Estado nutricional de pacientes renais crônicos submetidos a tratamento hemodialítico em um hospital de referência de Pernambuco. Nutr Clín Diet Hosp [Internet]. 2017 [acesso em 2020 Nov 12];37(3):58-65. doi: 10.12873/373brunosoares

10. Ikizler TA, Burrowes JD, Byham-Gray LD, Campbell KL, Carrero JJ, Chan W, et al. KDOQI Clinical Practice Guidelines for Nutrition in Chronic Kidney Disease: 2019 Update. Am J Kidney Dis [Internet]. 2020 [acesso em 2020 Nov 12];76(3, Supl 1):S1-S107. doi: https://doi.org/10.1053/j.ajkd.2020.05.006

11. Kalantar-Zadeh K, Kleiner M, Dunne E, Lee GH, Luft FC. A modified quantitative subjective global assessment of nutrition for dialysis patients. Nephrol Dial Transplant [Internet]. 1999 [acesso em 2020 Nov 12];14(7):1732-8. doi: 10.1093/ndt/14.7.1732

12. Kamimura MA, Avesani CM. Métodos compostos de avaliação nutricional. In: Cuppari L, Avesani CM, Kamimura MA. Nutrição na Doença Renal Crônica. Barueri: Manole; 2013. p.199-212.

13. World Health Organization. Physical Status: the use and interpretation of anthropometry. WHO Technical Report Series n 854. Geneva, Swi: World Health Organization; 1995.

14. Callaway CW, Chumlea WC, Bouchard C, Himes JH, Lohman TG, Martin AD, et al. Circumference. In: Lohman TG, Roche AF, Martorel R. Anthropometrics standartization reference manual. Champaign: Human Kinetics Books; 1988.

15. Harrison GG, Buskirk ER, Carter JEL, Johnston FE, LohmanTG, Pollock MR, et al. Skinfold thicknesses and measurement technique. In: Lohman TG, Roche AF, Martorel R. Anthropometrics standartization reference manual. Champaign: Human Kinetics Books; 1988.

16. Oliveira CMC, Kubrusly M, Mota RS, Choukroun G, Brandão JB Neto, Silva CABS. Adductor Pollicis Muscle Thickness: a promising anthropometric parameter for patients with chronic renal failure. J Ren Nutr [Internet]. 2012 [acesso em 2020 Nov 12];22(3):307-16. doi: 10.1053/j.jrn.2011.07.006

17. Lopes MB, Silva LF, Lopes GB, Penalva MA, Matos CM, Robinson BM, et al. Additional contribution of the Malnutrition-Inflammation Score to predict mortality and patient-reported outcomes as compared with its components in a cohort of African descent hemodialysis patients. J Ren Nutr [Internet]. 2017 [acesso em 2020 Nov 12];27(1):45-52. doi: 10.1053/j.jrn.2016.08.006

18. Sampaio GC, Brito ACD, Barros AJF, Hortegal EV, Dias RSC. Inflamação e associação com parâmetros clínicos e nutricionais de pacientes em hemodiálise. Ciênc Saúde [Internet]. 2017 [acesso em 2020 Nov 12];10(2):71-76. doi: 10.15448/1983-652X.2017.2.24244.

19. Castro AS, Pessoa PP, Barroso CF, Araújo GN, Sousa MP, Oliveira DL, et al. Zinco e risco cardiovascular de pacientes nefropatas em tratamento de hemodiálise. Rev Bras Promoç Saúde [Internet]. 2018 [acesso em 2020 Nov 12];31(1):1-7. doi: 10.5020/18061230.2018.5803

20. Lemos LB, Moraes GS, Lemos GS, Nery AA. Automedicação em pacientes renais crônicos hemodialíticos. Rev Bras Promoç Saúde [Internet]. 2020 [acesso em 2020 Nov 12];33:9906. doi: 10.5020/18061230.2020.9906

21. Ministério da Saúde (BR), Secretaria de Vigilância em Saúde, Departamento de Análise de Situação de 
Saúde. Plano de ações estratégicas para o enfrentamento das doenças crônicas não transmissíveis (DCNT) no Brasil 2011-2022. Brasília: Ministério da Saúde; 2011.

22. Bandeira SBC, Cansanção K, Paula TP, Peres WAF. Evaluation of the prognostic significance of the malnutrition inflammation score in hemodialysis patients. Clin Nutr ESPEN [Internet]. 2020 [acesso em 2020 Nov 12];35:109-115. doi: 10.1016/j.clnesp.2019.10.019

23. Rambod M, Bross R, Zitterkoph J, Benner D, Pithia J, Colman S, et al. Association of MalnutritionInflammation Score with quality of life and mortality in hemodialysis patients: a 5-year prospective cohort study. Am J Kidney Dis [Internet]. 2009 [acesso em 2020 Nov 12];53(2):298-309. doi: 10.1053/j. ajkd.2008.09.018

24. Alvarenga LA, Andrade BD, Moreira MA, Nascimento RP, Macedo ID, Aguiar AS. Análise do perfil nutricional de pacientes renais crônicos em hemodiálise em relação ao tempo de tratamento. J Bras Nefrol [Internet]. 2017 [acesso em 2020 Nov 12];39(3):283-6. doi: 10.5935/0101-2800.20170052

25. Moorthi RN, Avin KG. Clinical relevance of sarcopenia in chronic kidney disease. Curr Opin Nephrol Hypertens [Internet]. 2017 [acesso em 2020 Nov 12];26(3):219-28. doi: 10.1097/MNH.0000000000000318

26. Machado AD, Rissotto CM, Martins CTB, Bazanelli AP. Associação entre o consumo energético e proteico e a espessura do músculo adutor do polegar em pacientes em hemodiálise. Ciênc Saúde [Internet]. 2017 [acesso em 2020 Nov 12];10(1):3-9. doi: 10.15448/1983-652X.2017.1.24111

27. Howden EJ, Coombes JS, Isbel NM. The role of exercise training in the management of chronic kidney disease. Curr Opin Nephrol Hypertens [Internet]. 2015 [acesso em 2020 Nov 12];24(6):480-7. doi:10.1097/ MNH.0000000000000165

28. Huang M, Lv A, Wang J, Xu N, Ma G, Zhai Z. Exercise Training and Outcomes in Hemodialysis Patients: Systematic Review and Meta-Analysis. Am J Nephrol [Internet]. 2019 [acesso em 2020 Nov 12];50(4):240-54. doi: 10.1159/000502447

29. Martins ECV, Pereira VFS, Sales OS, Pereira PAL. Tempo de hemodiálise e o estado nutricional em pacientes com doença renal crônica. Braspen J. 2017;32(1):54-57.

\section{Endereço do primeiro autor:}

Jéssica Almeida Leite

Faculdade de Nutrição - Departamento de Alimentos e Nutrição

Universidade Federal de Mato Grosso

Av. Fernando Corrêa da Costa, 2367, Bloco CCBSI

Bairro Boa Esperança

CEP: 78060-900 - Cuiabá - MT - Brasil

E-mail: jessica_leite96@hotmail.com

\section{Endereço para correspondência:}

Gabriela Dalcin Durante

Faculdade de Nutrição - Departamento de Alimentos e Nutrição

Universidade Federal de Mato Grosso

Av. Fernando Corrêa da Costa, 2367, Bloco CCBSI

Bairro Boa Esperança

CEP: 78060-900 - Cuiabá - MT - Brasil

E-mail: gabrielad.durante@gmail.com

Como citar: Leite JA, Sousa PO, Borges MY, Rodrigues PRM, Beserra BTS, Durante GD. Análise dos fatores associados ao risco nutricional de pacientes em hemodiálise. Rev Bras Promoç Saúde. 2021;34:11271. 